\title{
A Note on the Population Statistics of Electron Avalanches and Streamers
}

\author{
T. Ficker
}

Ultraviolet tests focused on population statistics of electron avalanches were not included in our earlier work [1], [2]. Now they have been performed, and it is shown that a recently derived statistical pattern fits all the measured data very well.

Keywords: Population statistics, electron avalanches, fractal statistical pattern, ultraviolet data.

\section{Introduction}

Our recently published paper [1] presented a new generalized statistical pattern for the population distribution of electron avalanches and streamers. The pattern includes the Fury and Pareto distributions, both of which have been used for sparsely and highly populated avalanches, respectively. The pattern, i.e. the probability density function, has the following form

$$
\begin{aligned}
& F(n)=\frac{1}{\bar{n}} \sum_{j=0}^{J}(\bar{K} \cdot \bar{N})^{j} \cdot\left[1-\frac{\bar{N}}{\bar{n}}\right]^{n-1}, \\
& \bar{n}=\exp \left[\int_{0}^{x} \alpha\left(x^{\prime}\right) \mathrm{d} x^{\prime}\right], \\
& D=\frac{\ln (\bar{K})}{\ln (\bar{N})}
\end{aligned}
$$

where $\bar{K}, \bar{N}$ and $J$ are fitting parameters whose physical meaning has been explained in detail in [1] . Quantity $D$ is the so-called fractal dimension and its value $(0 \leq D \leq 3)$ is a "measure' of the space delocalization of avalanche pulsating discharges in the gap between electrodes.

Numerous tests have been performed to verify the functionality of pattern (1). The tests employed both electrical [1] and optical [2] data measured in our laboratory. All these types of tests have confirmed that the generalized pattern (1) can fit the data quite reliably. However, tests of one type were still missing, i.e., tests employing ultraviolet (UV) experimental data. The purpose of this short note is to present such tests.

\section{Ultraviolet tests}

UV-pulses coming from avalanches when they are crossing the discharge gap provide a unique opportunity to count their repetition rates and intensities. A convenient instrument for this purpose is a photomultiplier working in the UV-region. A method employing photomultipliers for registrating of ultraviolet radiation accompanying collisional ionization within avalanches allows us to analyze electron components independently from those of ion.

The experimental arrangement is shown in Fig. 1. The photomultiplier is located near the discharge gap, its signal is amplified and transferred to the digitizer. The files of the sta-

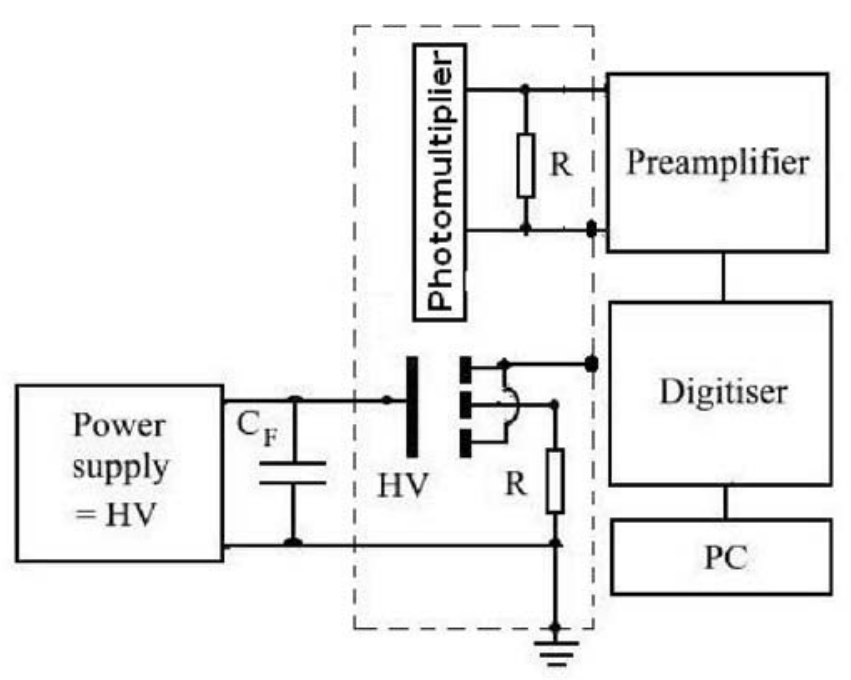

Fig. 1: Experimental arrangement for capturing of UV-pulses

tistical data captured in the digitizer are processed off-line in a computer.

Recently, extensive measurements of 'UV-statistics' have been performed in our laboratory and one typical example of these measurements is shown in Fig. 2. We can observe very good behavior of the fractal pattern (1) when comparing it with experimental data. UV-pulses were captured in the form of voltage pulses acquired across the resistance $R=100 \mathrm{k} \Omega$. Since we did not calibrate the voltage pulses $u$ against the number of electrons, our resulting distribution curves $F(u)$ were dependent on $u$ instead of $n$. When assuming linear proportionality $u \approx$ const $\cdot n$, the curves $F(u)$ will preserve the same shapes as $F(n)$, i.e. they will both possess the same value of fractal dimension $D$. The quantity $g$ in Fig. 2 seemingly has a similar purpose as a normalization constant, i.e. $w(u)=g \cdot f(U)$, but instead of the normalization correction it represents a 'shift' between unnormalized data and the unnormalized distribution function $F(u)$.

\section{Conclusion}

The results of the analysis of ultraviolet pulses have unmistakably shown that the fractal pattern (1) is a convenient approximation of the population statistics of electron avalanches. This conclusion is also in full agreement with the electrical [1] and optical [2] measurements performed earlier. 


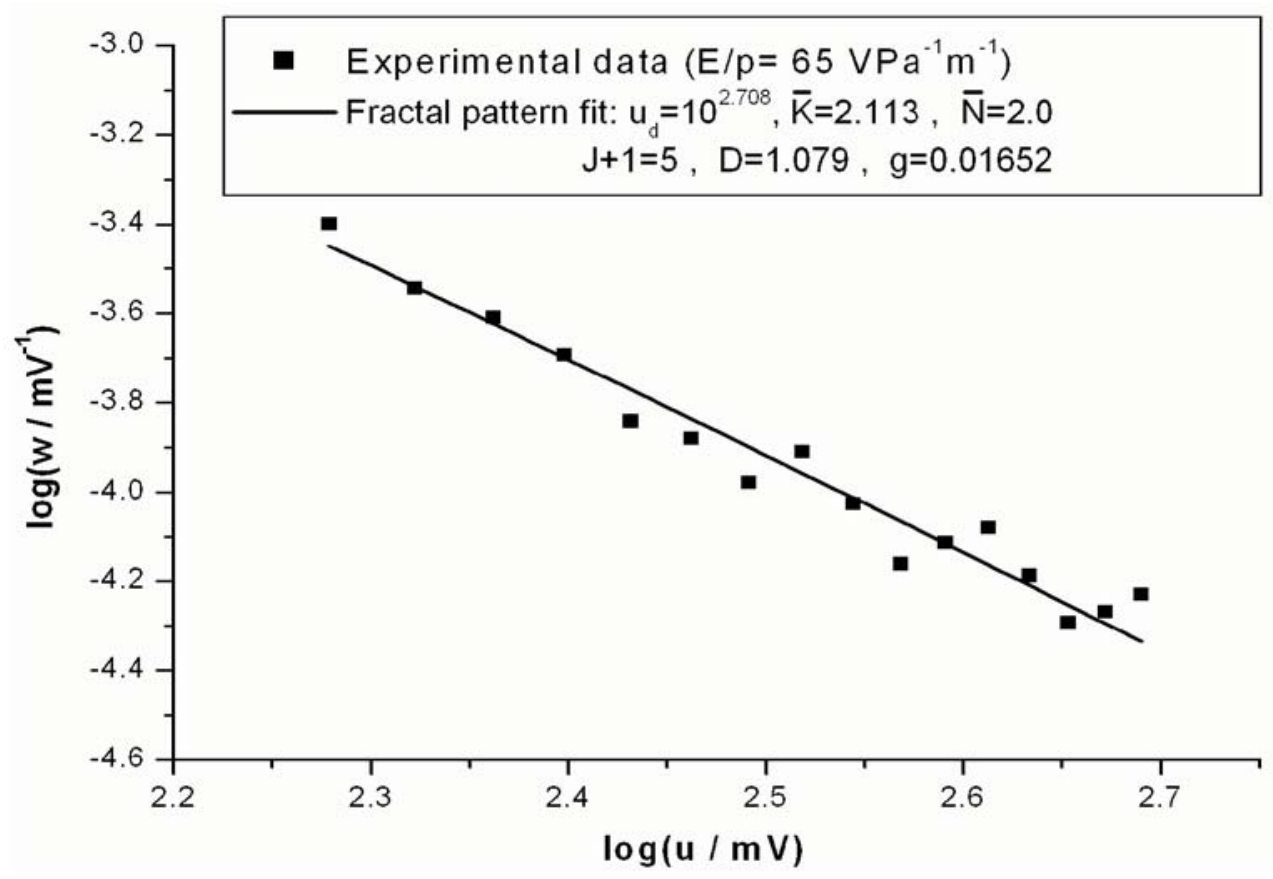

Fig. 2: Fractal pattern fitting UV-data

\section{Acknowledgments}

This work has been supported by the Grant Agency of the Czech Republic under Grant no. 202/07/1207.

\section{References}

[1] Ficker, T.: Acta Polytechnica, Vol. 47 (2007), p. 31.

[2] Ficker, T.: IEEE Transactions on Dielectrics and Electrical Insulations, Vol. 11 (2004), p. 136.

Prof. RNDr. Tomáš Ficker, DrSc.

phone: +420541 147661

e-mail: ficker.t@fce.vutbr.cz

Department of Physics

Faculty of Civil Engineering

University of Technology

Veveří 95

66237 Brno, Czech Republic 